\title{
In vivo Impedance Imaging with Total Variation Regularization
}

\author{
A. Borsic, B. M. Graham, A. Adler, W. R. B. Lionheart
}

\begin{abstract}
We show that electrical impedance tomography (EIT) image reconstruction algorithms with regularization based on the Total Variation (TV) functional are suitable for in vivo imaging of physiological data. This reconstruction approach helps to preserve discontinuities in reconstructed profiles, such as step changes in electrical properties at inter-organ boundaries, which are typically smoothed by traditional reconstruction algorithms. The use of the TV functional for regularization leads to the minimization of a non-differentiable objective function in the inverse formulation. This cannot be efficiently solved with traditional optimization techniques such as the Newton Method. We explore two implementations methods for regularization with the TV functional: the Lagged Diffusivity method and the Primal Dual Interior Point Method (PD-IPM). First we clarify the implementation details of these algorithms for EIT reconstruction. Next, we analyze the performance of these algorithms on noisy simulated data. Finally, we show reconstructed EIT images of in-vivo data for ventilation and gastric emptying studies. In comparison to traditional quadratic regularization, $T V$ regularization shows improved ability to reconstruct sharp contrasts.
\end{abstract}

Keywords: Electrical Impedance Tomography, EIT, Regularization, Total Variation, TV, Lagged Diffusivity, Primal Dual Interior Point Method

\section{INTRODUCTION}

Electrical Impedance Tomography (EIT) uses surface electrodes to make measurements from which an image of the electrical conductivity distribution within some medium is computed. The inverse conductivity problem is ill-posed [1]; consequently regularization techniques have been adopted in order to stabilize the inversion. Most common regularization methods impose (explicitly or implicitly) a penalty on nonsmooth regions in a reconstructed image. Such methods confer stability to the reconstruction process, but limit the capability of describing sharp variations in the sought parameter.

One technique to permit image regularization without imposing smoothing is the Total Variation (TV) formulation of regularization. The Total Variation functional is assuming an important role in the regularization of inverse problems belonging to many disciplines, thanks to its ability to preserve discontinuities in the reconstructed profiles. Edge preservation, if it works successfully, has clear clinical benefits. The cases explored in this work include images of the lungs and stomach, which have well defined organ boundaries that present sharp

Copyright (c) 2009 IEEE. Personal use of this material is permitted. However, permission to use this material for any other purposes must be obtained from the IEEE by sending a request to pubs-permissions@ieee.org. A. Borsic is with the Thayer School of Engineering, Dartmouth College, USA, email: Andrea.Borsic@Dartmouth.edu, B. M. Graham is with the School of Information Technology and Engineering, University of Ottawa, Canada, A. Adler is with the Department of Systems and Computer Engineering, Carleton University, Canada, W. R. B. Lionheart is with the School of Mathematics, University of Manchester, Manchester, UK transitions in conductivity. The ability thus of reconstructing sharp changes should lead to a better estimation of the boundaries and also to a better accuracy in the estimated values. For example, in the case of lung imaging, sharp boundaries can occur within a diseased lung, at the interface between ventilated and collapsed regions. Sharp images allow therefore to better identify the boundary between the two regions. Other applications of EIT that might benefit from sharp reconstructions are those related to cancer detection. In these applications, where EIT has the potential to be used as a screening tool, it is important to detect small localized conductivity contrasts. Quadratic algorithms blur such contrasts and make more difficult to estimate the size and the contrast value, due to the partial volume effect that occurs. Sharper reconstructions would allow better detection.

To date, almost all in-vivo studies use quadratic regularization or backprojection algorithms, which are known to blur image contrasts.

Edge preserving algorithms like the ones that use TV regularization have not been used as they are more complex to implement and thought not to be robust enough. In this paper we develop a normalized time-difference EIT image reconstruction using TV regularization, and show that it works well for imaging of in-vivo experimental data of lung ventilation (normal and diseased) and gastric emptying. In this work we use time difference EIT for reconstructing the change in conductivity between data frames. This method is widely used to alleviate systematic errors from instrument and electrode positioning inaccuracies in medical applications [2].

In Section II we introduce the general problem of EIT reconstruction, we introduce quadratic regularization and the properties of the TV functional. In Section III we discuss the practical problems that TV regularization poses in terms of non-differentiability and we introduce the Lagged Diffusivity and the Primal-Dual Interior Point Method algorithms, which we apply to impedance tomography. In Section IV we evaluate the proposed methods on syntectic and on in-vivo data, showing the characteristics of TV regularized images and that TV regularization can be applied successfully to clinical data. In Section V we draw conclusions regarding the work and results presented in this manuscript.

\section{IMAGE RECONSTRUCTION}

In EIT a set of electrodes are attached to the patient, currents are passed through the electrodes and the resulting potentials are measured. By using a numerical model of the experiment the conductivity is reconstructed as a least squares fitting problem. 


\section{A. Forward Model}

The forward problem is modeled with a low-frequency approximation, where the electric field is considered conservative and the conduction currents dominant with respect to the displacement currents, which leads to the following partial differential equation:

$$
\nabla \cdot \sigma \nabla u=0 \quad \text { on } \Omega
$$

where $\sigma$ is the conductivity or admittivity of the body to be imaged, $u$ is the electric potential, and $\Omega$ the body to be imaged. Electrodes are modeled with boundary conditions that in the EIT field are referred to as Complete Electrode Model [3]. The electrode model accounts for the contact impedance by stating that for each portion of the boundary $\partial \Omega_{\ell}$ underneath electrode $\ell$ :

$$
u+z_{c} \sigma \frac{\partial u}{\partial \vec{n}}=V_{\ell} \quad \text { on } \partial \Omega_{\ell} \quad \ell=1 \ldots \mathrm{L}
$$

where $z_{c}$ is the contact impedance, $\ell$ is the electrode number, $V_{\ell}$ is the potential resulting at electrode $\ell$, and $\mathrm{L}$ is the number of electrodes. For each electrode the flux of the current density through the contact surface must equal the injected current:

$$
\int_{\partial \Omega_{\ell}} \sigma \frac{\partial u}{\partial \vec{n}}=I_{\ell} \quad \text { on } \partial \Omega_{\ell} \quad \ell=1 \ldots \mathrm{L}
$$

where $I_{\ell}$ is the injected current at electrode $\ell$.

Underneath the inter-electrode gaps no current should flow, and the following condition is applied:

$$
\frac{\partial u}{\partial \vec{n}}=0 \quad \text { on } \partial \Omega \backslash\left\{\partial \Omega_{1} \cup \ldots \cup \partial \Omega_{\mathrm{L}}\right\}
$$

\section{B. Parameter Estimation}

We consider difference imaging, where the goal of the reconstruction is to recover the conductivity difference, due to physiological changes, that have occurred in the region of interest during a certain time interval. This approach is common in medical applications of EIT, as it reduces different types of image artifacts, for example breathing artifacts and movement artifacts [2].

In difference imaging, a first data set of voltages $\boldsymbol{V}\left(\sigma_{1}\right)$ is acquired at time $t_{1}$ corresponding to a reference conductivity distribution $\sigma_{1}$, a second set of voltage data $\boldsymbol{V}\left(\sigma_{2}\right)$ is acquired at time $t_{2}$, corresponding to a conductivity distribution $\sigma_{2}$. The vector of data difference is formed as:

$$
\mathbf{d}=\boldsymbol{V}\left(\sigma_{2}\right)-\boldsymbol{V}\left(\sigma_{1}\right)
$$

The goal is to reconstruct the conductivity change $\Theta=$ $\sigma_{2}-\sigma_{1}$ with respect to the reference conductivity $\sigma_{1}$, by predicting with the forward model the simulated data $\boldsymbol{W}(\Theta)$ corresponding to a change in conductivity:

$$
\boldsymbol{W}(\Theta)=\boldsymbol{V}\left(\sigma_{1}+\Theta\right)-\boldsymbol{V}\left(\sigma_{1}\right)
$$

and fitting the model to the data in the least-squares sense:

$$
\Theta_{\text {rec }}=\arg \min _{\Theta} \frac{1}{2}\|\boldsymbol{W}(\Theta)-\mathbf{d}\|^{2}+\alpha G(\Theta)
$$

The estimation is regularized with a Tikhonov style regularization, where $\alpha$ is the Tikhonov factor, and $G(\Theta)$ is the regularization functional that stabilizes the inversion. As we consider difference measurements, the conductivity change $\Theta$ with respect to the reference $\sigma_{1}$ can take both positive and negative values, so (6) is an unconstrained minimization problem.

\section{Quadratic Regularization Functionals}

In practice the forward model is implemented with a Finite Element Method or, less commonly, with a Finite Difference Method, and the conductivity is thus discretized. In this context the functional $G(\Theta)$ is often assumed to be of the form:

$$
G(\boldsymbol{\Theta})=\|\mathbf{L} \Theta\|^{2}
$$

where we indicate with the bold character $\Theta$ the vector of discrete conductivity values and where $\mathbf{L}$ is a regularization matrix. In the literature there are several choices for the matrix $\mathbf{L}$, for example the identity matrix [4], a positive diagonal matrix [5], approximations of first and second order differential operators [6], and the inverse of a Gaussian matrix [7]. Algorithms of this class fall into general framework:

$$
\boldsymbol{\Theta}_{\text {rec }}=\arg \min _{\boldsymbol{\Theta}} \frac{1}{2}\|\boldsymbol{W}(\boldsymbol{\Theta})-\mathbf{d}\|^{2}+\alpha\|\mathbf{L} \boldsymbol{\Theta}\|^{2}
$$

The framework expressed by eq (9) can be called quadratic regularization since the 2-norm is used. A norm guarantees that the regularization functional is always non-negative, as a penalty term should be, and more important, in the case of the 2-norm, the resulting functional is differentiable, leading to well embellished and easy to implement solutions of the minimization problem. Quadratic regularization, because of its differentiability, has been the common framework for solving several inverse problems, and particularly for EIT [4],[5],[6],[8],[9].

In practical implementations the optimization problem (9) is solved iteratively by using the Gauss Newton method, leading to a conductivity update:

$\delta \boldsymbol{\Theta}_{k}=-\left(\mathbf{J}_{k}^{T} \mathbf{J}_{k}+\alpha \mathbf{L}^{T} \mathbf{L}\right)^{-1}\left[\mathbf{J}_{k}^{T}\left(\boldsymbol{W}\left(\boldsymbol{\Theta}_{k}\right)-\mathbf{d}\right)-\alpha \mathbf{L}^{T} \mathbf{L} \Theta_{k}\right]$

where $k$ is the iteration number, $\delta \boldsymbol{\Theta}_{k}$ is the conductivity update at iteration $k$, and $J_{k}$ is the Jacobian of the forward operator $\boldsymbol{W}(\boldsymbol{\Theta})$ computed for $\Theta=\boldsymbol{\Theta}_{k}$. The drawback in using quadratic regularization schemes is that, regardless of the choice of $\mathbf{L}$, the technique cannot reconstruct step changes, as smooth solutions are favored.

\section{Total Variation Regularization Functional}

There are situations in almost every field of application of EIT where the imaged conductivity has discontinuities. In the medical field an example is that of the inter organ boundaries where each organ has its own electrical properties. Several approaches have been investigated in order to overcome these limitations. Often they can be considered a way to introduce prior information. An example is anisotropic regularization [10][11] where the structure of the expected sudden changes is assumed to be roughly known. The smoothness constraints are relaxed therefore in the direction normal to the discontinuities. 
In this way the algorithm better describes rapid variations in the object, however prior structural information needs to be known in order to adopt such methods. Recently combination of level set methods and TV regularization have been proposed as well for elliptic inverse problems [12] and specifically for EIT [13]. These methods can be used where the imaged body is known a priori to have regions of constant conductivity.

In many least squares formulations (9), regularization matrices $\mathbf{L}$ are discrete representations of differential operators and are used in conjunction with the 2-norm. A different approach is represented by the choice of the total variation functional, which is still a differential operator but leads to a $L^{1}$ regularization scheme. The total variation (TV) of a conductivity image $\Theta$ is defined as:

$$
T V(\Theta)=\int_{\Omega}|\nabla \Theta| d \Omega
$$

where $\Omega$ is the region to be imaged.

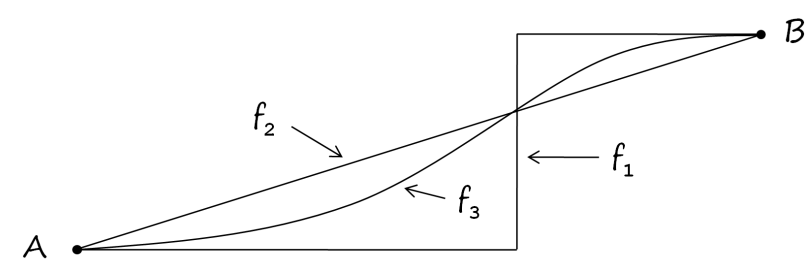

Fig. 1. Two points $A$ and $B$ connected by three different monotonically increasing functions $\left(f_{1}, f_{2}, f_{3}\right)$, all of them have the same Total Variation.

The TV functional was first employed by Rudin [14] for regularizing the restoration of noisy images. The effectiveness of the method in recovering discontinuous images can be understood by examining the following one dimensional situation.

Suppose that the two points A and B of Figure 1 are connected by a path. Three possible functions $f(x)$ connecting them are shown. As the functions are monotonically increasing, the TV of each is:

$$
T V(f)=\int_{A}^{B} f^{\prime}(x) d x=f(B)-f(A)
$$

which is the same value for each function. TV treats $f_{1}, f_{2}$ and $f_{3}$ in the same way and when used as a penalty term in a Tikhonov regularized inverse problem, will not bias the result towards a smooth solution. On the other hand, the $L^{2}$ norm yields different values for $f_{1}, f_{2}$ and $f_{3}$. When used as a penalty term the $L^{2}$ norm will bias the solution towards smoother functions, for which the $L^{2}$ norm assumes smaller values. Another way to understand the differences with other techniques is to consider the discretized version of equation (11). In our implementation we describe the conductivity as piecewise constant, using a $2 \mathrm{D}$ finite element mesh. Being the conductivity constant over each FEM element, $\nabla \Theta$ is non-null only on the edges between elements, where the conductivity presents a step change from one element to the other. For the $i$-th edge, shared by the FEM elements $m(i)$ and $n(i)$, the jump in conductivity is: $\left|\boldsymbol{\Theta}_{m(i)}-\boldsymbol{\Theta}_{n(i)}\right|$. The variation over the whole image can be found by integrating this jump over all the edges of the mesh:

$$
T V(\boldsymbol{\Theta})=\sum_{i} l_{i}\left|\boldsymbol{\Theta}_{m(i)}-\boldsymbol{\Theta}_{n(i)}\right|
$$

where $l_{i}$ is the length of the $i$-th edge in the mesh, $m(i)$ and $n(i)$ are the indices of the two elements sharing the $i$-th edge, and the index $i$ ranges over all the edges. Equation (13) can be expressed in terms of matrices as:

$$
T V(\boldsymbol{\Theta})=\sum_{i}\left|\mathbf{L}_{i} \boldsymbol{\Theta}\right|
$$

where $\mathbf{L}$ is a sparse matrix, with one row per each edge in the mesh. Every row $\mathbf{L}_{i}$ has two non zero elements in the columns $m(i)$ and $n(i): \mathbf{L}_{i}=\left[0, \ldots, 0, l_{i}, 0, \ldots, 0,-l_{i}, 0 \ldots 0\right]$. TV regularization is therefore of the $L^{1}$ kind: it is a sum of absolute values. The absolute value guarantees the positivity of the penalty function but unfortunately results in non-differentiability in the points where $\boldsymbol{\Theta}_{m(i)}=\boldsymbol{\Theta}_{n(i)}$. The numerical problem thus needs to be addressed properly. However, the important gain is that the $L^{1}$ regularization does not penalize image discontinuities.

\section{Solving THE TV REgularized EIT Problem}

Two different approaches have been proposed in the past for application of TV regularization to EIT, the first by Rudin [14] and Dobson [15] and the second by Somersalo [16] and by Kolehmainen [17]. Rudin and Dobson use a deterministic framework and replace the absolute value function in the neighborhood of zero by a polynomial to obtain a continuously differentiable function. Somersalo and Kolehmainen apply Markov Chain Monte Carlo (MCMC) methods to solve the TV regularized inverse problem. Probabilistic methods, such as MCMC, offer central estimates by sampling the posterior probability density of the sought parameters and therefore differentiability is not required (smoothing of the TV functional). Though this method is successful, the sampling process involves a substantial computational effort and the image reconstruction can be lengthy. Deterministic methods on the other hand require differentiability and a number of different approaches has been developed.

Deterministic numerical methods have been used for the regularization of image de-noising and restoration problems with the TV functional. The numerical efficiency and stability are the main issues to be addressed. Most of the deterministic methods draw from ongoing operations research advances, as TV minimization belongs to the important classes of problems known as "minimization of sum of norms" [18] and "linear $L^{1}$ problems" [19] .

Recent developments in operations research [18] have provided new classes of methods to deal efficiently with the problems of minimizing the sum of absolute values. Chan [20] has drawn from these advances and investigated the problem of restoring images with Primal Dual-Interior Point Methods (PD-IPM). In this paper we introduce and compare the performance of two methods: the "Lagged Diffusivity" 
method, which is straightforward to implement but requires smoothing of the TV functional to avoid numerical instability, and an algorithm based on the PD-IPM theory, which is more efficient and has no stability problems.

\section{A. Methods Based on Approximations of TV}

Early algorithms for TV regularization in image denoising applications approximate the TV functional in different manners in order to remove the non-differentiability. This is achieved by substituting the absolute value with a function that has a smooth corner near the origin of the axes and that approximates the absolute value function elsewhere [21] [22] [23] [24] [25] [26], [17]. In the following we define $T V_{\beta}(\Theta)$ as

$$
T V_{\beta}(\Theta)=\int_{\Omega} \sqrt{|\nabla \Theta|^{2}+\beta}
$$

for $\beta>0$ the functional is differentiable and $T V_{\beta}(\Theta) \rightarrow$ $T V(\Theta)$ for $\beta \rightarrow 0$. We formulate the $\mathrm{TV}_{\beta}$ regularized inverse problem as

$$
\Theta_{\text {rec }}=\arg \min _{\Theta} \frac{1}{2}\|\boldsymbol{W}(\Theta)-\mathbf{d}\|^{2}+\alpha T V_{\beta}(\Theta)
$$

and when the conductivity is discretized this becomes

$$
\boldsymbol{\Theta}_{r e c}=\arg \min _{\boldsymbol{\Theta}} \frac{1}{2}\|\boldsymbol{W}(\boldsymbol{\Theta})-\mathbf{d}\|^{2}+\alpha \sum_{i} \sqrt{\left|\mathbf{L}_{i} \boldsymbol{\Theta}\right|^{2}+\beta}
$$

where $\mathbf{L}$ is as in equation 14. In order to minimize the objective function in (16), the Newton's Method has been considered in several works [22], [24], [25]. In this case the iterative update equation becomes:

$$
\begin{aligned}
& \delta \boldsymbol{\Theta}_{k}=-\left(\mathbf{J}_{k}^{T} \mathbf{J}_{k}+\alpha \mathbf{L}^{T} \mathbf{E}_{k}^{-1} \mathbf{F}_{k} \mathbf{L}\right)^{-1} \\
& \cdot\left[\mathbf{J}_{k}^{T}\left(\boldsymbol{W}\left(\boldsymbol{\Theta}_{k}\right)-\mathbf{d}\right)-\alpha \mathbf{L}^{T} \mathbf{E}_{k}^{-1} \mathbf{L} \boldsymbol{\Theta}_{k}\right]
\end{aligned}
$$

with

$$
\begin{array}{r}
\eta_{i}=\sqrt{\left|\mathbf{L}_{i} \boldsymbol{\Theta}_{k}\right|^{2}+\beta} \\
\mathbf{E}_{k}=\operatorname{diag}\left(\eta_{i}\right) \\
\mathbf{F}_{k}=\operatorname{diag}\left(1-\frac{\left|\mathbf{L}_{i} \boldsymbol{\Theta}_{k}\right|^{2}}{\eta_{i}^{2}}\right)
\end{array}
$$

The convergence properties of the Newton's Method have been studied by Vogel [27] and by Chan [24] in the context of image denoising problems. Both authors report that the method results convergent only for values of $\beta$ which are sufficiently large; in other words the method has a small convergence region, with respect to $\beta$. Vogel reports that for small values of $\beta$ the size of the convergence region can be shown to be proportional to $\beta^{3 / 2}$, therefore decreasing with beta decreasing. The same difficulties have been found in [28] in the context of EIT. It is obviously desirable to solve (17) for small values of $\beta$ for approximating a true TV regularization, but this is difficult in practice.

Noting that the approximated Hessian matrix in (18) is

$$
\mathbf{J}_{k}^{T} \mathbf{J}_{k}+\alpha \mathbf{L}^{T} \mathbf{E}_{k}^{-1} \mathbf{F}_{k} \mathbf{L}
$$

and that, by expanding $\mathbf{F}_{k}$, this can be written as

$$
\mathbf{J}_{k}^{T} \mathbf{J}_{k}+\alpha \mathbf{L}^{T} \mathbf{E}_{k}^{-1} \mathbf{L}-\alpha \mathbf{L}^{T} \mathbf{E}_{k}^{-1} \operatorname{diag}\left(\frac{\left|\mathbf{L}_{i} \boldsymbol{\Theta}_{k}\right|^{2}}{\eta_{i}^{2}}\right) \mathbf{L}
$$

it has been proposed [22] to drop the negative semidefinite matrix

$$
-\mathbf{L}^{T} \mathbf{E}_{k}^{-1} \operatorname{diag}\left(\frac{\left|\mathbf{L}_{i} \Theta_{k}\right|^{2}}{\eta_{i}^{2}}\right) \mathbf{L}
$$

leading to the update equation

$$
\begin{gathered}
\delta \boldsymbol{\Theta}_{k}=-\left(\mathbf{J}_{k}^{T} \mathbf{J}_{k}+\alpha \mathbf{L}^{T} \mathbf{E}_{k}^{-1} \mathbf{L}\right)^{-1} . \\
\cdot\left[\mathbf{J}_{k}^{T}\left(\boldsymbol{W}\left(\boldsymbol{\Theta}_{k}\right)-\mathbf{d}\right)-\alpha \mathbf{L}^{T} \mathbf{E}_{k}^{-1} \mathbf{L} \boldsymbol{\Theta}_{k}\right]
\end{gathered}
$$

This method, sometimes referred to as "Lagged Diffusivity" was used by Vogel [22] and Dobson [23] as an alternative to Newton's Method. The method is believed to have better convergence properties with respect to Newton Method thanks to dropping the negative semidefinite matrix from the Hessian. The method is globally convergent, though local convergence is only linear [23]. As discussed in the remainder of the present work, we have implemented this method for comparison purposes with respect to the primal dual - interior point algorithm introduced next. Though the Lagged Diffusivity method shows better convergence properties than the Newton's Method, still it converges slowly or becomes unstable for small values of $\beta$ [28]. Continuation strategies are required for starting the algorithm with larger values of $\beta$ and progressively and carefully reducing $\beta$ as the algorithm progresses.

\section{B. Primal Dual - Interior Point Methods}

An efficient method for dealing with the nondifferentiability of the TV regularization functional has been proposed by Chan [20] in the context of image denoising applications. The method is based on the primal-dual theory developed by Andersen [18].

We will label as primal $(\mathrm{P})$ the TV regularized inverse problem formulation

$$
(P) \quad \arg \min _{\boldsymbol{\Theta}} \frac{1}{2}\|\boldsymbol{W}(\boldsymbol{\Theta})-\mathbf{d}\|^{2}+\alpha \sum_{i}\left|\mathbf{L}_{i} \boldsymbol{\Theta}\right|
$$

noting that for each $i$

$$
\left|\mathbf{L}_{i} \boldsymbol{\Theta}\right|=\max _{\chi_{i}:\left|\chi_{i}\right| \leq 1} \chi_{i} \mathbf{L}_{i} \boldsymbol{\Theta}
$$

where $\chi$ is a vector of scalar auxiliary variables. By applying (27) to $(P)$, a second, equivalent problem, which is a maximization problem, is obtained

$$
\text { (D) } \max _{\boldsymbol{\chi}:\left|\boldsymbol{\chi}_{i}\right| \leq 1} \min _{\boldsymbol{\Theta}} \frac{1}{2}\|\boldsymbol{W}(\boldsymbol{\Theta})-\mathbf{d}\|^{2}+\alpha \sum_{i} \boldsymbol{\chi}_{i} \mathbf{L}_{i} \boldsymbol{\Theta}
$$

this problem is called dual (D) and the auxiliary variables $\chi$ are labeled dual variables. For feasible points [18] of the dual problem (i.e. $\left|\chi_{i}\right| \leq 1$ ) the objective function of (P) can be shown to take greater values than the objective function of the dual problem (D). The two objective functions take the same value at a single point, which is the optimal point both for (P) and for (D). Such optimal point can therefore be sought by nulling the difference between the two objective functions, 
called the primal-dual gap:

$$
\begin{array}{r}
\frac{1}{2}\|\boldsymbol{W}(\boldsymbol{\Theta})-\mathbf{d}\|^{2}+\alpha \sum_{i}\left|\mathbf{L}_{i} \boldsymbol{\Theta}\right|- \\
+\frac{1}{2}\|\boldsymbol{W}(\boldsymbol{\Theta})-\mathbf{d}\|^{2}-\alpha \sum_{i} \boldsymbol{\chi}_{i} \mathbf{L}_{i} \boldsymbol{\Theta} \\
=\alpha \sum_{i}\left(\left|\mathbf{L}_{i} \boldsymbol{\Theta}\right|-\boldsymbol{\chi}_{i} \mathbf{L}_{i} \boldsymbol{\Theta}\right)
\end{array}
$$

The primal-dual (PD) gap is null if for each $i$ either $\mathbf{L}_{i} \Theta=0$ or $\chi_{i}=\left|\mathbf{L}_{i} \Theta\right| /\left(\mathbf{L}_{i} \Theta\right)$. The condition that nulls the PD gap is therefore

$$
\left(\left|\mathbf{L}_{i} \boldsymbol{\Theta}\right|-\chi_{i} \mathbf{L}_{i} \boldsymbol{\Theta}\right)=0 \quad i=1, \ldots, n
$$

and it is called complementarity condition. The complementarity condition captures the optimality of both (P) and (D). An important class of algorithms, called Primal-Dual Interior Point Methods (PD-IPM) is based on a framework that enforces the complementarity condition, the primal feasibility (if any) and the dual feasibility (if any) [18] [29].

The PD-IPM framework for the TV regularized EIT inverse problem can be written noticing that 28 can be simplified by enforcing the condition

$$
\mathbf{J}(\boldsymbol{\Theta})^{T}[\boldsymbol{W}(\boldsymbol{\Theta})-\mathbf{d}]+\alpha \mathbf{L} \boldsymbol{\Theta}=\mathbf{0}
$$

which is the first order condition for the inner minimization problem on $\Theta$, and thus (D) can be rewritten as:

$$
\text { (D) } \max _{\boldsymbol{\chi}:\left|\chi_{i}\right| \leq 1} \frac{1}{2}\|\boldsymbol{W}(\boldsymbol{\Theta})-\mathbf{d}\|^{2}+\alpha \sum_{i} \boldsymbol{\chi}_{i} \mathbf{L}_{i} \Theta
$$

with $\boldsymbol{\Theta}: \mathbf{J}(\boldsymbol{\Theta})^{T}[\boldsymbol{W}(\boldsymbol{\Theta})-\mathbf{d}]+\alpha \mathbf{L} \boldsymbol{\Theta}=\mathbf{0}$

The PD-IPM framework is written thus enforcing the complementarity condition for (P) and (D) and by maintaining the feasibility conditions, obtaining:

$$
\begin{gathered}
\left|\boldsymbol{\chi}_{i}\right| \leq 1 \quad i=1, \ldots, n \\
\mathbf{J}(\boldsymbol{\Theta})^{T}[\boldsymbol{W}(\boldsymbol{\Theta})-\mathbf{d}]+\alpha \mathbf{L} \boldsymbol{\Theta}=\mathbf{0} \\
\left(\left|\mathbf{L}_{i} \boldsymbol{\Theta}\right|-\boldsymbol{\chi}_{i} \mathbf{L}_{i} \boldsymbol{\Theta}\right)=0 \quad i=1, \ldots, n
\end{gathered}
$$

The systems of equations (33), (34), (35) constitutes the framework and needs to be solved jointly on $(\Theta, \chi)$. Here as well the absolute value in (35) needs to be smoothed similarly to (15) in order to obtain differentiability, though the meaning of this smoothing is different in the context of the PD-IPM framework than in (15). This is done by replacing $\left|\mathbf{L}_{i} \Theta\right|$ by $\sqrt{\left|\mathbf{L}_{i} \boldsymbol{\Theta}\right|^{2}+\beta}$ in (35). The smoothed feasibility condition is called the centering condition [18] and it leads to a smooth pair of optimization problems $\left(P_{\beta}\right)$ and $\left(D_{\beta}\right)$. The effect of such condition is that solutions are approached, as $\beta$ is decreased, from points away from the the boundary $\chi:\left|\chi_{i}\right|=1$ of the feasible region, from which the name of centering condition and of the notion of interior point methods.

The Gauss Newton method can now be applied to solve smoothed primal-dual problem, obtaining the following system for the updates $\delta \Theta$ and $\delta \chi$ of the primal and dual variables:
$\left[\begin{array}{cc}\mathbf{J}_{k}^{T} \mathbf{J}_{k} & \alpha \mathbf{L}^{T} \\ \mathbf{K}_{k} \mathbf{L} & -\mathbf{E}_{k}\end{array}\right]\left[\begin{array}{c}\delta \boldsymbol{\Theta}_{k} \\ \delta \boldsymbol{\chi}_{k}\end{array}\right]=-\left[\begin{array}{c}\mathbf{J}_{k}^{T}\left(\boldsymbol{W}\left(\boldsymbol{\Theta}_{k}\right)-\mathbf{d}\right)+\alpha \mathbf{L}^{T} \boldsymbol{\chi}_{k} \\ \mathbf{L} \Theta_{k}-\mathbf{E}_{k} \boldsymbol{\chi}_{k}\end{array}\right]$

with

$$
\mathbf{K}_{k}=\operatorname{diag}\left(1-\frac{\boldsymbol{\chi}_{i}^{(k)} L_{i} \boldsymbol{\Theta}_{k}}{\eta_{i}}\right)
$$

Equation (36) leads to the following update for the primal variables:

$$
\begin{gathered}
\delta \boldsymbol{\Theta}_{k}=-\left[\mathbf{J}_{k}^{T} \mathbf{J}_{k}+\alpha \mathbf{L}^{T} \mathbf{E}_{k}^{-1} \mathbf{K}_{k} \mathbf{L}\right]^{-1} . \\
\cdot\left[\mathbf{J}_{k}^{T}\left(\boldsymbol{W}\left(\boldsymbol{\Theta}_{k}\right)-\mathbf{d}\right)+\alpha \mathbf{L}^{T} \mathbf{E}_{k}^{-1} \mathbf{L} \boldsymbol{\Theta}_{k}\right]
\end{gathered}
$$

and the following for the dual variables:

$$
\delta \chi_{k}=-\chi_{k}+\mathbf{E}_{k}^{-1} \mathbf{L} \Theta_{k}+\mathbf{E}_{k} \mathbf{K}_{k} \mathbf{L} \delta \Theta_{k}
$$

Equations (38) and (39) can therefore be applied iteratively to solve the non-linear inversion (26). In our work we start the iterative procedure by initializing the primal variables with a single step of the quadratic reconstruction, and the dual variables to $\chi_{0}=\mathbf{0}$, which is a feasible point for the dual variables.

Some care must be taken on the dual variable update, to maintain dual feasibility. A traditional line search procedure [30] with feasibility checks is not suitable as the dual update direction is not guaranteed to be an ascent direction for the modified dual objective function $\left(D_{\beta}\right)$.

The simplest way to compute the update is called the scaling rule [18] which is defined to work as follows

$$
\chi^{(k+1)}=\varphi^{*}\left(\chi^{(k)}+\delta \chi\right)
$$

where $\varphi^{*}$ is a scalar value such that

$$
\varphi^{*}=\sup \left\{\varphi: \varphi\left|\chi_{i}^{(k)}+\delta \chi_{i}\right| \leq 1, \quad i=1, \ldots, n\right\}
$$

An alternative way is to calculate the exact step length to the boundary, applying what is called the step length rule [18]

$$
\chi^{(k+1)}=\chi^{(k)}+\min \left(1, \varphi^{*}\right) \delta \chi
$$

where $\varphi^{*}$ is a scalar value such that

$$
\varphi^{*}=\sup \left\{\varphi:\left|\chi_{i}^{(k)}+\varphi \delta \chi_{i}\right| \leq 1, \quad i=1, \ldots, n\right\}
$$

In the context of EIT, and in tomography in general, the computation involved in calculating the exact step length to the boundary of the dual feasibility region is negligible compared to the whole algorithm iteration. It is convenient therefore to adopt the step length rule, which in our experiments resulted in a better convergence. The scaling rule has the further disadvantage of always placing $\chi$ on the boundary of the feasible region, which prevents the algorithm from following the central path (sequence of points in the interior of the feasibility region that converges to the optimal point). Concerning the updates on the primal variable, the update direction $\delta \Theta$ is a descent direction for $\left(P_{\beta}\right)$ therefore a line search procedure could be appropriate. In our numerical experiments we have found that for relatively small contrasts (e.g. 3:1) the primal line search procedure is not needed, as 
the steps are unitary. For larger contrasts a line search on the primal variable guarantees the stability of the algorithm.

As a side note it interesting to observe that at the first iteration, with the initialization $\chi_{0}=\mathbf{0}$, the matrix $K$ results equal to the identity and therefore the update for the primal variable (38) is equal to the update of the Lagged Diffusivity method (18). As the algorithm progresses, the matrix $K$ tends to the matrix $F$ of the Newton's method, therefore the PrimalDual Interior point method can be seen as an interpolation between the Lagged Diffusivity, away from the optimal point, and the Newton method, which has quadratic convergence, but only in a neighborhood of the optimal point.

\section{Evaluation Procedure}

In order to evaluate the properties of TV regularized algorithms we have implemented the Lagged Diffusivity (we will label this algorithm as "LD") and the Primal Dual Interior Point Method (we will label this algorithm as "PD") and tested them against a traditional quadratic reconstruction algorithm as in (9) (we will label this algorithm as "QR"). Tests were conducted on in vivo data and on simulated data. The in vivo data is time-difference data, collected with 16 electrode EIT systems implementing the adjacent protocol [7]. Though we introduced a fully non-linear framework in the previous sections, we use for reconstruction of medical data a linearized version of the algorithms. The choice of using a linearized reconstruction is common in EIT medical timedifference applications: the not perfectly known geometry of the patient, the not perfectly known position of the electrodes and the time-changing contour of the imaging region contribute significant errors to measured data. These errors are typically much greater than those resulting from linearizing the forward operator. We linearize therefore the forward operator as $\boldsymbol{V}\left(\sigma_{1}+\Theta\right) \approx \boldsymbol{V}\left(\sigma_{1}\right)+\mathbf{J} \Theta$, where $\mathbf{J}$ is the Jacobian computed for $\sigma=\sigma_{1}$. From this follows that $\boldsymbol{W}(\Theta) \approx \mathbf{J} \Theta$ and the general formulation of the inverse problem (7) becomes:

$$
\Theta_{\text {rec }}=\arg \min _{\Theta} \frac{1}{2}\|\mathbf{J} \Theta-\mathbf{d}\|^{2}+\alpha G(\Theta)
$$

Equations (9) (16) and (26) change accordingly. The quadratic problem can be solved with a single step of (10) using $\mathbf{J}_{k}=\mathbf{J}\left(\sigma_{1}\right)$, while the LD and PD algorithms remain iterative as (18) and (38) (39) depend on the conductivity distribution through the matrices $\mathbf{E}$ and $\mathbf{K}$. The TV regularized problem is solved iterating respectively (18) and (38) (39) and maintaining $\mathbf{J}_{k}=\mathbf{J}\left(\sigma_{1}\right)$. As the reference conductivity $\sigma_{1}$ is not known, we linearized all the reconstructions around the uniform conductivity $\sigma_{1}=1 \mathrm{Sm}^{-1}$. Naturally this procedure reconstructs conductivities in an arbitrary scale and not in absolute values, but this is a common, accepted, effective method for reconstructing time-difference data. The non-linear framework we provide remains valid for the reconstruction of absolute images, using $\boldsymbol{W}(\Theta)=\boldsymbol{V}(\Theta)$.

In order to evaluate the algorithms, simulated data was generated as well, as discussed in the following subsections.

\section{A. Simulated Data}

We consider a 16 electrode EIT system using adjacent stimulation and measurement (this matches the experimental measurements we consider in the next subsection). A simulated phantom was generated using a 2-dimensional mesh with 1024 first order triangular elements (see Figure 2), where electrodes are described with the Complete Electrode Model. The distribution of the simulated conductivity that has been used for the tests provides sharp inclusions and also provides a challenge for the reconstruction as there is a narrow gap between the two inclusion regions. The simulated phantom has a background conductivity of $1 \mathrm{Sm}^{-1}$, in the bottom part of the phantom there is a larger inclusion with a value of $1.5 \mathrm{Sm}^{-1}$, in the top part of the phantom there is a smaller inclusion with a conductivity value of $0.5 \mathrm{Sm}^{-1}$. Time-difference data were simulated by computing simulated data on a reference distribution (homogenous phantom with a conductivity of 1 $\mathrm{Sm}^{-1}$ ) and by taking the difference of such data with the data computed on the phantom with the conductivity distribution just described and shown in Figure 2. Images are reconstructed on a two dimensional mesh with 576 elements not matching the mesh used for forward computations, in order to avoid what is referred as an inverse crime [31]. In addition to this, in order to simulate systematic and random errors in the data acquisition process, a synthetic noise vector was generated, this to be added to the data in different noise levels. Such vector $\nu$, with the same length as the data difference vector $\mathbf{d}$, was generated extracting each sample from a Normal distribution with zero mean and standard deviation of 1 , and then normalized as:

$$
\boldsymbol{\nu}=\boldsymbol{\nu} \frac{\operatorname{std}(\mathbf{d})}{\operatorname{std}(\boldsymbol{\nu})}
$$

where $\operatorname{std}(\cdot)$ is the standard deviation of a vector. We specify noise levels in percentages, for example we indicate the noisy data produced as $\mathbf{d}+0.01 \boldsymbol{\nu}$ to have a $1 \%$ level of noise.

\section{B. Convergence Behavior}

We use a first series of numerical experiments for highlighting the typical convergence behavior of the LD and PD algorithms. As we have linearized the forward operator, the quadratic solution $\mathrm{QR}$ can be achieved with a single step of (10), while LD and PD remain iterative. The respective iterative update equations for $\mathrm{LD}$ and $\mathrm{PD}$, (18) and (38), depend on the products $\mathbf{L}^{T} \mathbf{E}_{k}^{-1} \mathbf{L}$ and $\mathbf{L}^{T} \mathbf{E}^{-1} \mathbf{K} \mathbf{L}$, where the diagonal matrix $\mathbf{E}^{-1}$ and the diagonal matrix $\mathbf{E}^{-1} \mathbf{K}$ depends on $\eta_{i}$, and therefore on the reconstructed conductivity itself. For this reason the reconstruction remains iterative. The value of the diagonal elements of these matrices is decreasing for increasing values of $\eta_{i}$ (See eq. (20) and (37)). This weights less larger jumps of conductivity between neighboring pixels. This is a feedback effect, from the reconstructed conductivity distribution to the regularization, and, similarly to dynamic systems we refer to it as "dynamic regularization". Due to this, the LD and PD algorithms need a number of iterations for both the conductivity distribution and the matrices $\mathbf{E}$ and $\mathbf{K}$ to settle, as these are interlinked. In our simulations we 
adopt a stopping criterion based on the relative decrease of the objective functional:

$$
H(\Theta)=\frac{1}{2}\|\mathbf{J} \Theta-\mathbf{d}\|^{2}+\alpha T V(\boldsymbol{\Theta})
$$

The iterative algorithms are stopped when the relative decrease in the functional is less than $1 \%$, that is:

$$
\left[\frac{H\left(\boldsymbol{\Theta}_{k+1}\right)}{H\left(\boldsymbol{\Theta}_{k}\right)}-1\right] \leq 0.01
$$

where $k$ is the iteration number. With this stopping criterion the LD algorithm converges in 8 iterations and the PD algorithm in 10. In the top row of Figure 3 are shown the $2^{\text {nd }}$, the $5^{\text {th }}$ and the $10^{t h}$ reconstruction steps for the synthetic difference data for the PD algorithm. The bottom row shows the same reconstruction steps for the LD algorithm. All the sub-figures are represented on the same color scale. While the $2^{\text {nd }}$ and $5^{\text {th }}$ step for both the algorithms shows similar reconstructed images, the images at the $10^{\text {th }}$ step are different. The LD algorithm does not sharpen significantly the image as iterations progress, while the $10^{t h}$ iteration of the PD algorithm shows a much stronger sharpening effect. This is as well represented by a higher value of $\|L \Theta\|^{2}$, reported in the captions of Figure 3, which reaches $4.99 \times 10^{-1}$ for the $\mathrm{LD}$ algorithm and $8.56 \times 10^{-1}$ for PD. The term $\|L \Theta\|^{2}$, as discussed before, assumes larger values for fast spatial variations, and it is thus indicative of sharp images. The Total Variation of the images, on the other hand, measures the variations present in the image, but now how sharp they are, and for this reason it can lead to sharper images if used as a regularization term. The $\mathrm{PD}$ algorithm results in a TV of 3.08, compared to 3.28 for the LD algorithm. The better performance of PD can be attributed to the use of a much smaller value of $\beta$ than what possible with the LD algorithm, as discussed in the following sections.

In terms of computational effort, the LD and PD algorithms require a similar effort per iteration compared to quadratic algorithms. In the case of the LD algorithm the update equation (18) differs from the quadratic update (10) only for the presence of the matrices $\mathbf{E}^{-1}$ and $\mathbf{F}$, which are trivial to compute being diagonal. For both algorithms the computation of $\delta \boldsymbol{\Theta}_{k}$ by solving the dense linear system in (18) is dominant with respect to any other computation, and so the cost per iteration for the two algorithms is the same. In the case of the PD algorithm, there is both an update equation for the primal variables and one for the dual variables. Similarly to $L D$, the primal update (38) involves the diagonal matrices $\mathbf{E}^{-1}$ and $\mathbf{K}$, which are readily computed, and the main computational cost is represented by the solution of the dense linear system that gives $\delta \boldsymbol{\Theta}_{k}$. The dual update computation is quick, as it is formulated as a product of sparse matrices (39). From this analysis and in our numerical experience, the computational cost at each iteration of $\mathrm{QR}, \mathrm{LD}$ and $\mathrm{PD}$ is dominated by the solution of the dense linear systems in (10), (18), and (38) that give $\delta \Theta_{k}$, and this is identical for the three algorithms. The algorithms differ in the numbers of iterations that are required for reaching convergence. Typically LD requires a smaller number of iterations compared to PD. The first algorithm often converges in 7 to 10 iterations, while the second requires typically 10 to 14 iterations, in the cases we present. The PD algorithm takes more iterations to converge as it manages to significantly reduce the objective function for a larger number of steps, achieving thus shaper contrasts in the reconstructed images than the LD algorithm, as illustrated by the iterations in Figure 3.

\section{Noise Effects}

In order to study the noise performance of the algorithms, noise was added to the simulated data with noise levels of $0 \%, 1 \%, 2 \%$ and 3\%. Reconstructions are shown in Figure 4. All the sub-figures are represented on the same color scale. Columns are relative to the QR, LD and PD algorithms while rows are relative to different levels of noise. For the QR algorithm 1 iteration was used, as we are addressing the linearized problem. The termination criterion (47) was used for the LD and PD algorithms, and the number of iterations actually performed is reported in the caption of each subfigure. In terms of choice of Tikhonov factor, as the QR and $\mathrm{LD} / \mathrm{PD}$ algorithms present different regularization functionals, two different values were used for the $\mathrm{QR}$ algorithm and for the TV regularized algorithms. The two values were chosen automatically using the Fixed Noise Figure method [32], with a noise figure of 1 . This method finds the regularization parameter that results in a given amplification of noise in the reconstructed images. The Tikhonov parameter selected in this way results in the same noise amplification for different methods, which may then be fairly compared to each other.

Across all noise levels the differences between algorithms are noticeable and consistent. The PD algorithm produces the sharpest images, the QR algorithm the smoothest and the LD algorithm produces shaper images than the QR algorithm but not as sharp as PD. In the caption of each figure we report the value of $\|L \Theta\|^{2}$ and of $T V(\boldsymbol{\Theta})$. The quadratic functional $\|L \Theta\|^{2}$ is sensitive to the sharpness of the images, and presents larger values for the PD algorithm than for $\mathrm{LD}$ or $\mathrm{QR}$. This difference between LD and PD is due to the fact that the PD algorithm can be run with much smaller values of $\beta$ without incurring in stability problems [28]. In the simulations we used $\beta=10^{-3}$ for $\mathrm{LD}$ and $\beta=10^{-12}$ for PD. The smaller $\beta$ values the PD algorithm can be run with result in a better approximation of the TV functional and hence in the sharper images. We weren't able in our experiments to reduce the $\beta$ value for the LD to levels similar to the ones used for PD or anyway below $\beta=10^{-3}$, as this results in a slow convergence and eventually in instability. The PD-IPM framework is in fact known to be better than other schemes at dealing with nondifferentiability of the objective function, and this is a practical verification of this aspect. In each figure we report also the value of $T V(\boldsymbol{\Theta})$. As outlined in Figure 1, the Total Variation is not necessarily an indicator of the sharpness of the images: the PD reconstructions show smaller values for TV but the largest for $\|L \Theta\|^{2}$. This is a practical verification that the use of the TV functional allows reconstruction of sharp profiles, as $T V(\boldsymbol{\Theta})$ can take smaller values than $\|L \boldsymbol{\Theta}\|^{2}$ in the presence of sharp variations. 
For a noise level of $1 \%$ both the LD and PD algorithms show sharper reconstructions compared to QR, and PD is significantly shaper than LD. The TV regularized algorithms, and particularly PD, are capable of showing a better separation between the top and bottom inclusions, while the $\mathrm{QR}$ shows a smoother transition from the higher to the lower conductivity, offering a less clear separation between the inclusions. For a noise level of $2 \%$ the QR algorithm identifies the single top insulating inclusion as a double spot, while the TV regularized algorithms, and particularly PD, show a uniform spot. For a noise level of $3 \%$ for all the algorithms show artifacts, though the PD algorithms presents a more faithful reconstruction of the original image. In these images is more evident how the QR algorithm fails to identify the top insulating inclusion as a single spot. The TV algorithms describe better this source of contrast, this being a blocky distribution, matching well the TV prior. The PD algorithm offers a sharper reconstruction, compared the $\mathrm{LD}$ and $\mathrm{QR}$.

\section{In Vivo Data}

Three different sets of experimental data are considered. All EIT data were acquired with 16 electrodes arranged in a plane using an adjacent measurement scheme. The sets are relative to human gastric emptying (HGE), human breathing (HBR) and pig lung injury (PLI) studies. The HGE and HBR data were acquired using the system described in [33]. The PLI data were acquired using the system described in [34].

\section{E. Human Gastric Emptying}

Human gastric emptying data were collected for the study described in [35]. Briefly, electrodes were placed around the abdomen of a seated young male subject. Measured data were acquired before, $\boldsymbol{V}\left(\sigma_{1}\right)$, and after drinking, $\boldsymbol{V}\left(\sigma_{2}\right)$, Coca-Cola. The data have been reconstructed with the three algorithms QR, LD and PD, as illustrated in Figure 5. A mesh roughly resembling the anatomy of the subject was used, and all the three reconstruction algorithms identify clearly the change in conductivity that has occurred in the subject. The images show a localized negative impedance change, which is attributed to a local decrease in conductivity as the gas bubbles present in the drink fill the stomach of the subject. The three images are represented on the same color scale, for allowing comparison. The TV regularized $\mathrm{PD}$ and $\mathrm{LD}$ reconstructions show a greater sharpness for the localized conductivity change and are able to reconstruct a larger contrasts.

\section{F. Human Breathing}

Human breathing data were acquired from a healthy young male subject during normal breathing while seated. Electrodes were placed around the chest at the $6^{\text {th }}$ intercostal space. Measured data $\boldsymbol{V}\left(\sigma_{1}\right)$ and $\boldsymbol{V}\left(\sigma_{2}\right)$ corresponds to end-expiratory, and end-inspiratory cycles respectively. Images were reconstructed on a mesh roughly conforming to the anatomy of the subject, and results from the three different algorithms is shown in Figure 6. All the three algorithms identify the increased resistivity of the lungs due to the inspired air volume. Images are represented on the same color scale. Again reconstructions show the different characteristics of quadratic regularization with respect to total variation regularization, the second presenting sharper reconstructions and larger contrasts for conductivity distributions that present step changes.

\section{G. Pig Lung Injury}

Pig lung injury data were taken from the study of Frerichs [36]. Briefly, lung injury was induced by lavage of lungs of an anesthetized ventilated pig. Positive end-expiratory pressure (PEEP) was then progressively increased while EIT data were acquired. Two images are reconstructed. Data $\boldsymbol{V}\left(\sigma_{1}\right)$ and $\boldsymbol{V}\left(\sigma_{2}\right)$ are acquired at the end-expiratory and end-inspiratory cycles with a PEEP level of $0 \mathrm{~cm} \mathrm{H}_{2} \mathrm{O}$ for the first image and of $20 \mathrm{~cm} \mathrm{H}_{2} \mathrm{O}$ for the second image. Clinically it is known that supine subjects with injured lungs will receive less ventilation at the top of the lungs at low levels of PEEP and dependent lung regions will be recruited as PEEP increases. This physiology realistic effect shows with all the algorithms. The differences between the images are due to the shape of the reconstructed lung region. For the QR algorithm, a blurred region with a broad central peak is shown, while for the TV regularized algorithms, the lung regions appear as a more uniform image region with a flatter central plateau.

\section{DISCUSSION AND CONCLUSION}

In this work we present the use of Primal Dual - Interior Point Methods for efficiently using TV as a regularization functional in Electrical Impedance Tomography. Through numerical experiments and reconstruction of medical data we show that TV regularized algorithms produce sharper images compared to quadratic regularization algorithms and that these algorithms can be successfully applied to real data from medical experiments. For comparison purposes we implement two algorithms for TV regularized reconstruction: the Lagged Diffusivity algorithm and an algorithm based on the Primal Dual - Interior Point framework. The first algorithm, LD, requires minor modifications with respect to the common Gauss Newton quadratic scheme and the theoretical foundation of this method is readily understood as it stems from the application of the Gauss Newton method to the modified functional $\mathrm{TV}_{\beta}$. The Lagged Diffusivity method though, for small values of $\beta$, requires careful techniques for reducing and controlling the value of such parameter, as otherwise the method can become unstable or very slow to converge. This of course is an undesirable effect, and adds one layer of complexity to the actual implementation and use of the algorithm. We show that the second algorithm, PD, can be run with values of $\beta$ as small as $10^{-12}$ without requiring any control on $\beta$. This algorithm, based on the primal dual interior point framework, results thus in a better approximation of the TV functional, presenting sharper images, and has the important advantage of not requiring the control of an additional parameter: $\beta$ can simply be set to a small constant value that produces sharp images.

We have shown that TV regularization can be successfully applied to medical applications of EIT. The sharper 
reconstructed images and the fact that the technique presents larger contrasts for blocky conductivity distributions might result in enhancements in clinical applications. In particular we expect TV regularized algorithms to be useful, for example, in EIT cancer detection applications, where EIT is used for detecting localized high impedance tissues. An other area we TV regularized algorithms might provide enhancements over current techniques are lung imaging applications. The sharp impedance contrast between the lung and the surrounding tissue, or within an injured lung, where liquids might be present and provide a strong and sharp contrast, might be reconstructed better qualitatively and quantitatively by a TV regularized algorithm.

While the present work focuses on numerical methods and presents some in vivo results, we are interested in conducting future work in investigating the use of TV regularization on a wider scale and in different medical applications in order to gain a better understanding of the practical benefits that can be gained. An implementation of both the LD and PD algorithms has been released for public access with the EIDORS project (http://www.eidors.org/).

\section{REFERENCES}

[1] W. R. Breckon, "Image reconstruction in electrical impedance tomography," Ph.D. dissertation, Oxford Brookes Polytechnic, 1990.

[2] A. Adler, R. Guardo, and Y. Berthiaume, "Impedance imaging of lung ventilation: Do we need to account for chest expansion?" IEEE Trans. Biomed. Eng., vol. 43, pp. 414-420, 1996.

[3] E. Somersalo, M. Cheney, and D. Isaacson, "Existence and uniqueness for electrode models for electric current computed tomography," SIAM J Appl Math, vol. 52, pp. 1023-1040, 1992.

[4] T. J. Yorkey, J. G. Webster, and W. J. Tomkins, "An optimal impedance tomographic reconstruction algorithm," Proc. 8th Ann. Conf. IEEE Eng. Med. Biol. Soc., pp. 339-342, 1986.

[5] M. Cheney, D. Isaacson, J. C. Newell, S. Simake, and J. Goble, "NOSER: An algorithm for solving the inverse conductivity problem," Int J Imag Sys Technol, vol. 2, pp. 66-75, 1990.

[6] P. Hua, E. J. Woo, and J. G. Webster, "Iterative reconstruction methods using regularisation and optimal current patterns in elecrical impedance tomography," IEEE Trans. Med. Imag., vol. 10, pp. 621-628, 1991.

[7] A. Adler and R. Guardo, "Electrical impedance tomography: Regularized imaging and contrast detection," IEEE Trans Med Imgaging, vol. 15, pp. 170-179, 1996.

[8] 2nd EPSRC Engineering Network Meeting on Biomedical Applications of EIT., Ed., A Matlab Toolbox for the EIDORS Project to Reconstruct Two- and Three-Dimensional EIT Images, London, 2000.

[9] N. Polydorides and W. R. B. Lionheart, "A MATLAB based toolkit for three dimensional electrical impedance tomography: A contribution to the EIDORS project." Meas. Sci. Technol., vol. 13, pp. 1871-1883, 2002.

[10] J. P. Kaipio, V. Kolehmainen, M. Vauhkonen, and E. Somersalo, "Inverse problems with structural prior information," Inverse Problems, vol. 15, pp. 713-730, 1999.

[11] A. Borsic, W. R. B. Lionheart, and C. N. McLeod, "Generation of anisotropic-smoothness regularization filters for EIT," IEEE Transactions on Medical Imaging, vol. 21, no. 6, pp. 579-587, 2002.

[12] T. Chan and X. Tai, "Level set and total variation regularization for elliptic inverse problems with discontinuous coefficients," Journal of Computational Physics, vol. 193, no. 1, pp. 40-66, 2004.

[13] E. Chung, T. Chan, and X. Tai, "Electrical impedance tomography using level set representation and total variational regularization," Journal of Computational Physics, vol. 205, no. 1, pp. 357-372, 2005.

[14] L. I. Rudin, S. Osher, and E. Fatemi, "Nonlinear total variation based noise removal algorithms," Physica D, vol. 60, pp. 259-268, 1992.

[15] D. C. Dobson and F. Santosa, "Recovery of blocky images from noisy and blurred data," SIAM Journal on Applied Mathematics, vol. 56, no. 4, pp. 1181-1198, 1996.

[16] E. Somersalo, J. Kaipio, M. Vauhkonen, and D. Baroudi, "Impedance Imaging and Markov Chain Monte Carlo Methods," Proc. SPIE's 42nd Annual Meeting, pp. 175-185, 1997.
[17] V. Kolehmainen, "Novel approaches to image reconstruction in diffusion tomography," Ph.D. dissertation, Department of Applied Physics Kuopio University, 2001.

[18] K. D. Andersen, E. Christiansen, A. Conn, and M. L. Overton, "An efficient primal-dual interior-point method for minimizing a sum of Euclidean norms," SIAM J. on Scientific Computing, vol. 22, pp. 243$262,2000$.

[19] T. F. Coleman and Y. Li, "A globally and quadratically convergent affine scaling method for linear $l_{1}$ problems," SIAM J. on Optimization, vol. 3 , no. 3, pp. 609-629, 1992.

[20] T. Chan and P. Mulet, "Iterative methods for total variation restoration," UCLA CAM Report 96-38, 1996.

[21] R. Acar and C. R. Vogel, "Analysis of bounded variation penalty methods for ill-posed problems," Inverse Problems, vol. 10, pp. 12171229, 1994.

[22] C. R. Vogel and M. E. Oman, "Iterative methods for total variation denoising," SIAM J. Sci. Computing, vol. 17, pp. 227-238, 1996.

[23] D. C. Dobson and C. R. Vogel, "Convergence of an iterative method for total variation denoising," SIAM J. on Numerical Analysis, vol. 43, pp. 1779-1791, 1997.

[24] T. F. Chan, H. M. Zhou, and R. H. Chan, "A continuation method for total variation denoising problems," UCLA CAM Report 95-18, 1995.

[25] T. F. Chan, G. Golub, and P. Mulet, "A nonlinear primal dual method for TV-based image restoration," UCLA CAM Report 95-43, 1995.

[26] D. C. Dobson and F. Santosa, "An image enhancement technique for electrical impedance tomography," Inverse Problems, vol. 10, pp. 317334, 1994.

[27] C. R. Vogel, Nonsmooth Regularization, in H. W. Engl, A. K. Louis, and W. Rundell (Eds.), Inverse Problems in Geophysical Applications. SIAM, 1995.

[28] A. Borsic, "Regularisation methods for imaging from electrical measurements," Ph.D. dissertation, Oxford Brookes University, School of Engineering, 2002.

[29] S. J. Wright, Primal Dual Interior Point Methods. Philadelphia: SIAM, 1997.

[30] J. Nocedal and S. J. Wright, Numerical Optimization. Springer, 1999.

[31] A. Wirgin, "The inverse crime," Arxiv preprint math-ph/0401050, 2004.

[32] B. Graham and A. Adler, "Objective selection of hyperparameter for EIT," Physiological Measurement, vol. 27, no. 5, pp. 65-80, 2006.

[33] R. Guardo, C. Boulay, B. Murray, and M. Bertrand, "An experimental study in electrical impedance tomography using backprojection reconstruction," IEEE Transaction on Biomedical Engineering in Medicine and Biology Society, 2007. EMBS 2007. 29th Annual International Conference of the IEEE, vol. 43, p. 414:421, 1996.

[34] G. Hahn, T. Dudykevych, I. Frerichs, F. Thiel, and G. Hellige, "A high performance electrical impedance tomography (eit) system for clinical evaluation studies and space application," Proc. Conf. $2^{\text {nd }}$ European Medical and Biol. Eng. Vienna, Austria, 2002.

[35] A. Adler, R. Guardo, and Y. Berthiaume, "Imaging of gastric emptying with electrical impedance tomography," Canadian Medical and Biolological Eng. Soc., vol. 20, pp. 14-15, 1994.

[36] I. Frerichs, P. Dargaville, T. Dudykevych, and P. Rimensberger, "Electrical impedance tomography: a method for monitoring regional lung aeration and tidal volume distribution?" Intensive Care Medicine, vol. 29 , no. 12 , pp. 2312-2316, 2003. 


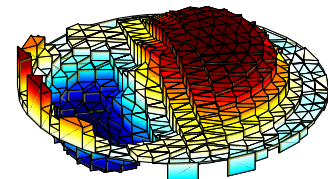

(a) LD iter=2,

$\|L \Theta\|^{2}=3.31 \times 10^{-1}$

$T V(\boldsymbol{\Theta})=3.61 \times 10^{0}$

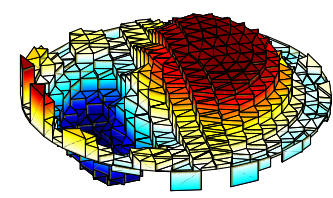

(d) PD iter $=2$,

$\|L \Theta\|^{2}=3.55 \times 10^{-1}$

$T V(\Theta)=3.99 \times 10^{\circ}$

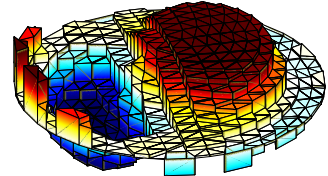

(b) LD iter $=5$,

$\|L \Theta\|^{2}=4.48 \times 10^{-1}$

$T V(\Theta)=3.33 \times 10^{\circ}$

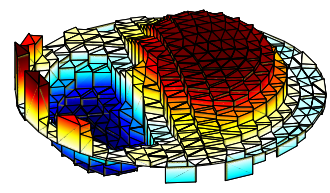

(e) $\mathrm{PD}$ iter $=5$,

$\|L \Theta\|^{2}=4.51 \times 10^{-1}$

$T V(\Theta)=3.55 \times 10^{\circ}$

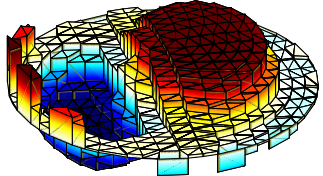

(c) LD iter $=10$,

$\|L \Theta\|^{2}=4.99 \times 10^{-1}$

$T V(\Theta)=3.28 \times 10^{0}$

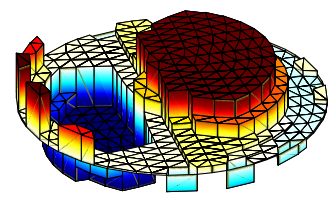

(f) $\mathrm{PD}$ iter $=10$,

$\|L \Theta\|^{2}=8.56 \times 10^{-1}$

$T V(\Theta)=3.08 \times 10^{0}$

Fig. 3. Convergence of the TV regularized algorithms. Different subfigures represent different steps of the reconstruction of the numerical phantom data. The top row illustrates the $2^{s} t, 5^{t} h$ and $10^{t} h$ steps for the Lagged Diffusivity algorithm, the bottom row the same iterative steps for the Primal Dual - Interior Point Method algorithm.

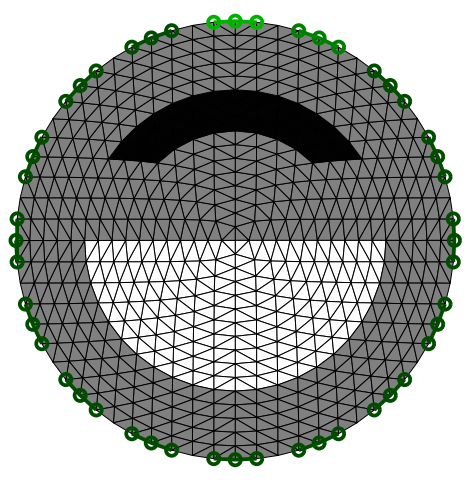

Fig. 2. Image of the numerical phantom used for generating the simulated data. The background conductivity value is $1 \mathrm{~S} \mathrm{~m}^{-1}$, the top inclusion presents a value of $0.5 \mathrm{~S} \mathrm{~m}^{-1}$ and the bottom inclusion a value of 1.5 $\mathrm{S} \mathrm{m}{ }^{-1}$. 


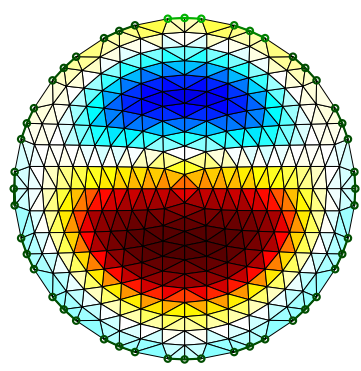

(a) $\mathrm{QR}, 0 \%$ noise, iter $=1$, $\|L \Theta\|^{2}=9.14 \times 10^{-4}$ $T V(\Theta)=7.12 \times 10^{-1}$

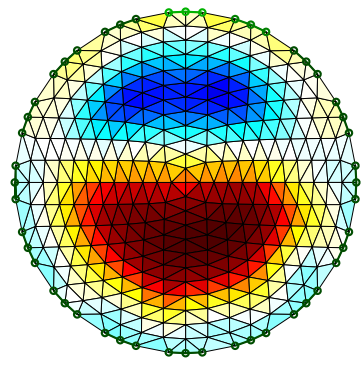

(d) QR, $1 \%$ noise, iter $=1$, $\|L \Theta\|^{2}=9.48 \times 10^{-4}$ $T V(\Theta)=7.28 \times 10^{-1}$

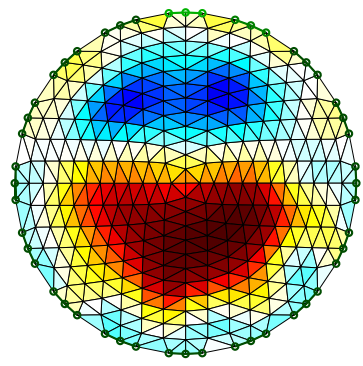

(g) $\mathrm{QR}, 2 \%$ noise, iter $=1$, $\|L \Theta\|^{2}=1.10 \times 10^{-3}$ $T V(\Theta)=7.87 \times 10^{-1}$

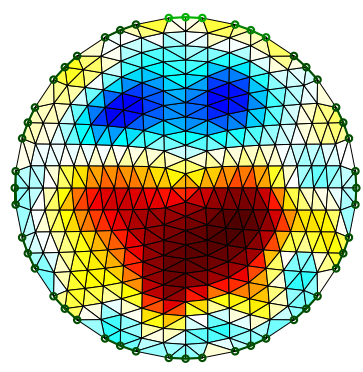

(j) QR, 3\% noise, iter $=1$, $\|L \Theta\|^{2}=1.38 \times 10^{-3}$
$T V(\Theta)=8.73 \times 10^{-1}$

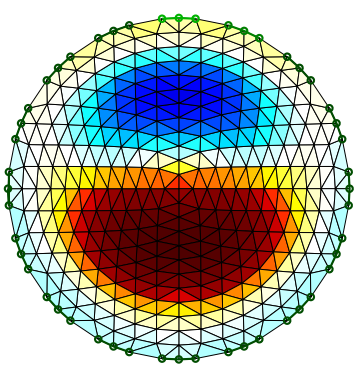

(b) LD, $0 \%$ noise, iter $=10$, $\|L \Theta\|^{2}=1.04 \times 10^{-3}$ $T V(\Theta)=6.09 \times 10^{-1}$

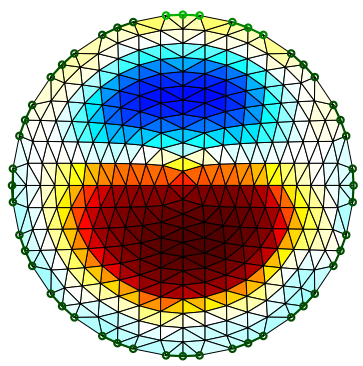

(e) $\mathrm{LD}, 1 \%$ noise, iter $=9$, $\|L \Theta\|^{2}=1.02 \times 10^{-3}$ $T V(\Theta)=6.11 \times 10^{-1}$

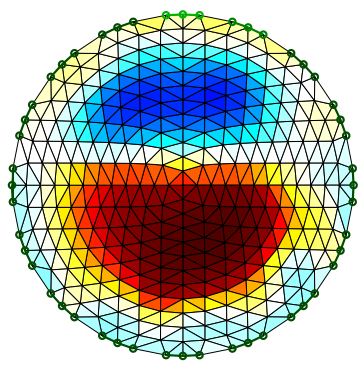

(h) LD, 2\% noise, iter $=8$, $\|L \Theta\|^{2}=1.08 \times 10^{-3}$ $T V(\Theta)=6.34 \times 10^{-1}$

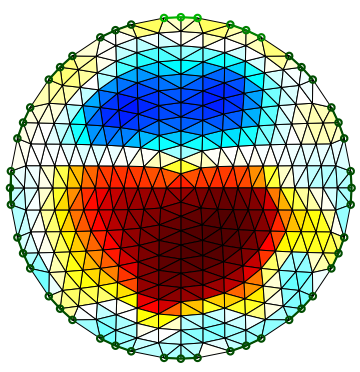

(k) $\mathrm{LD}, 3 \%$ noise, iter $=8$, $\|L \Theta\|^{2}=1.25 \times 10^{-3}$ $T V(\boldsymbol{\Theta})=6.77 \times 10^{-}$

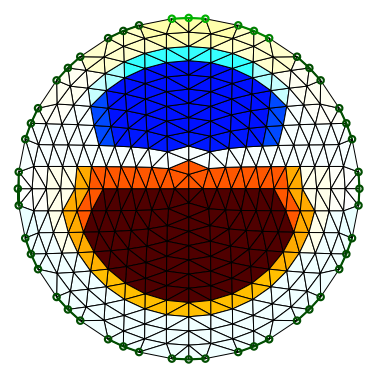

(c) $\mathrm{PD}, 0 \%$ noise, iter $=14$, $\|L \Theta\|^{2}=2.39 \times 10^{-3}$ $T V(\Theta)=4.87 \times 10^{-1}$

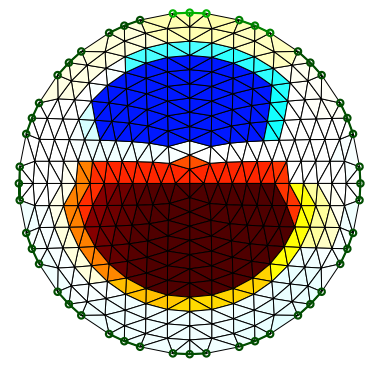

(f) $\mathrm{PD}, 1 \%$ noise, iter $=13$ $\|L \Theta\|^{2}=2.24 \times 10^{-3}$ $T V(\Theta)=4.86 \times 10^{-}$

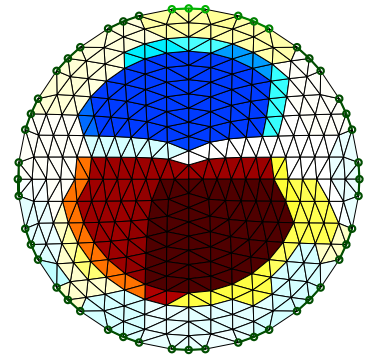

(i) $\mathrm{PD}, 2 \%$ noise, iter $=14$, $L \Theta \|^{2}=2.59 \times 10^{-3}$

$T V(\Theta)=5.07 \times 10^{-1}$

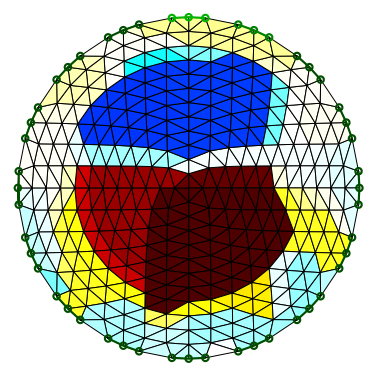

(1) $\mathrm{PD}, 3 \%$ noise, iter $=13$, $\|L \Theta\|^{2}=2.94 \times 10^{-3}$
$T V(\Theta)=5.47 \times 10^{-1}$

Fig. 4. Illustration of the noise performance of quadratic and TV regularized algorithms. Different subfigures represent reconstructions of the numerical phantom data for increasing noise levels and for different algorithms. The three columns show respectively the quadratic regularized algorithm, the Lagged Diffusivity Algorithm and the Primal Dual - Interior Point Method algorithm. Each row is relative to a different level of noise, from 0 to $3 \%$. All the figures are represented on the same color scale with a minimum value of $0.88 \mathrm{~S} \mathrm{~m}^{-1}$ and a maximum value of $1.18 \mathrm{~S} \mathrm{~m}^{-1}$. 


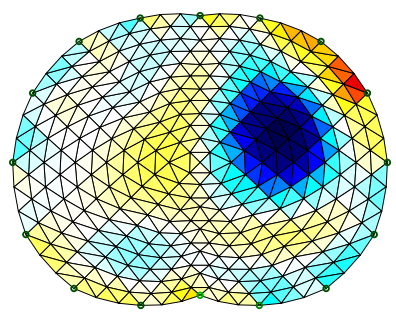

(a) QR, iter=1,

$\|L \Theta\|^{2}=9.57$

$T V(\Theta)=64.02$

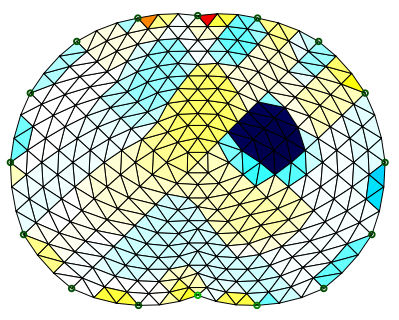

(b) LD, iter $=15$,

$\|L \Theta\|^{2}=204.6$

$T V(\Theta)=128.1$

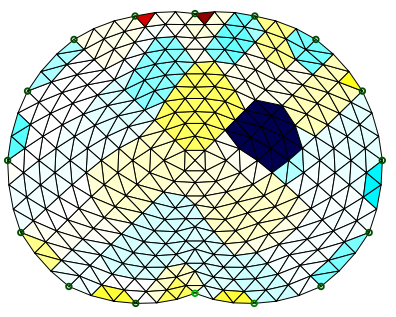

(c) PD, iter=11,

$\|L \Theta\|^{2}=305.3$

$T V(\Theta)=133.5$

Fig. 5. Time difference EIT image of human stomach emptying. Comparison of the quadratic and of the two TV regularized algorithms. All the figures are represented on the same color scale and in arbitrary conductivity units.

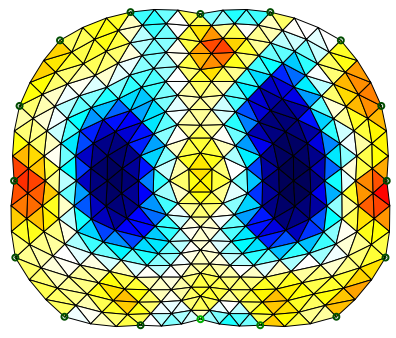

(a) $\mathrm{QR}$, iter $=1$,
$\|L \Theta\|^{2}=699.8$

$T V(\Theta)=628$

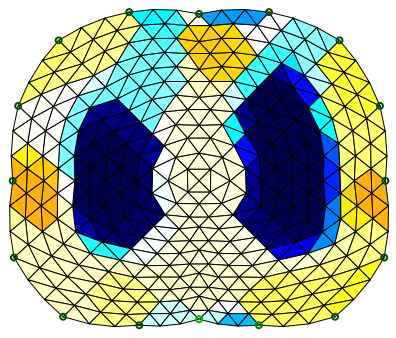

(b) $\mathrm{LD}$, iter $=18$,
$\|L \Theta\|^{2}=1667$

$T V(\Theta)=447.5$

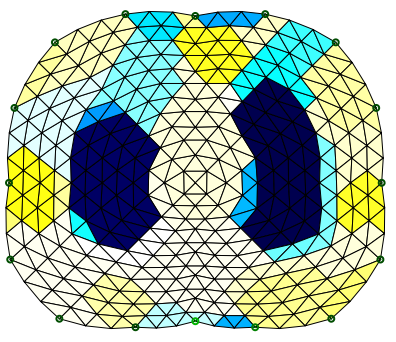

(c) PD, iter=12,

$\|L \Theta\|^{2}=2722$

$T V(\Theta)=475.7$

Fig. 6. Time difference EIT image of a human thorax during breathing, comparison of the quadratic and of the two TV regularized algorithms. All the figures are represented on the same color scale and in arbitrary conductivity units.

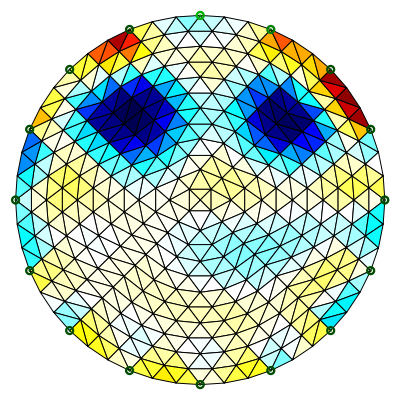

(a) $\mathrm{QR}$, iter $=1$,

$\|L \Theta\|^{2}=2.60 \times 10^{-5}$ $T V(\Theta)=1.01 \times 10^{-1}$

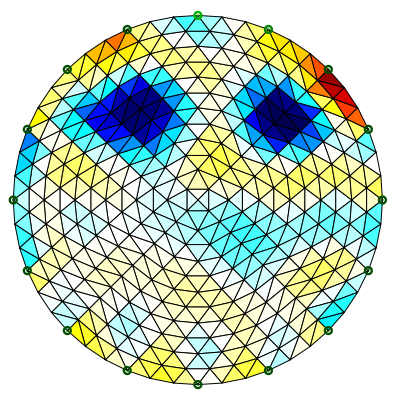

(b) $\mathrm{LD}$, iter $=4$,

$\|L \Theta\|^{2}=4.17 \times 10^{-5}$

$T V(\Theta)=1.28 \times 10^{-}$

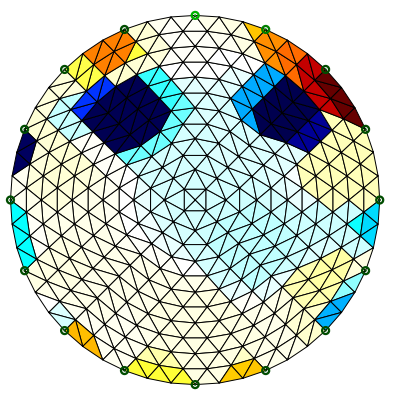

(c) PD, iter $=14$,

$\|L \Theta\|^{2}=7.79 \times 10^{-5}$

$T V(\Theta)=7.50 \times 10^{-2}$

Fig. 7. Time difference EIT image of injured swine lungs, PEEP $0 \mathrm{~cm} \mathrm{H}_{2} \mathrm{O}$. Comparison of the quadratic and the two TV regularized algorithms. All the figures are represented on the same color scale and in arbitrary conductivity units.

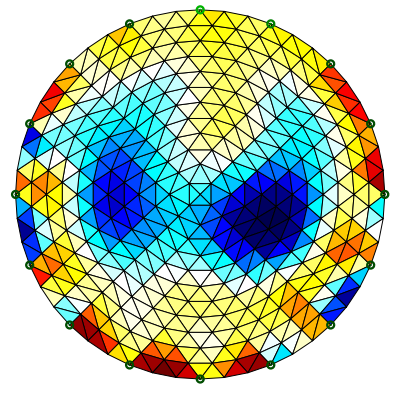

(a) $\mathrm{QR}$, iter $=1$ $\|L \Theta\|^{2}=1.16 \times 10^{-5}$
$T V(\Theta)=7.03 \times 10^{-2}$

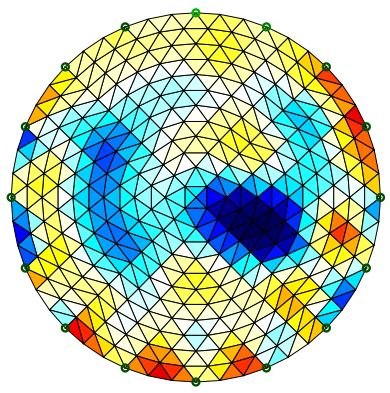

(b) $\mathrm{LD}$, iter $=3$, $\|L \Theta\|^{2}=1.89 \times 10^{-5}$ $T V(\Theta)=9.37 \times 10^{-2}$

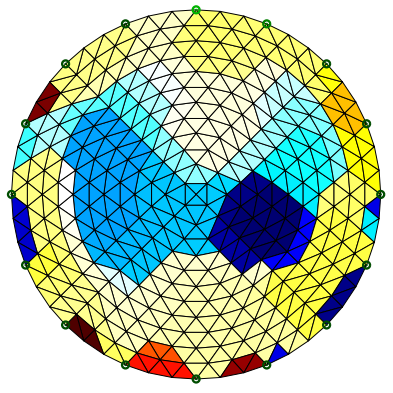

(c) $\mathrm{PD}$, iter $=15$ $\|L \Theta\|^{2}=2.77 \times 10^{-5}$ $T V(\Theta)=5.31 \times 10^{-2}$

Fig. 8. Time difference EIT image of injured swine lungs, PEEP $20 \mathrm{~cm} \mathrm{H}_{2} \mathrm{O}$. Comparison of the quadratic and of the two TV regularized algorithms. All the figures are represented on the same color scale and in arbitrary conductivity units. 